\title{
Gap Functions and Error Bounds for Set-Valued Vector Quasi Variational Inequality Problems
}

\author{
Rachana Gupta*, Aparna Mehra \\ Department of Mathematics, Indian Institute of Technology Delhi, Hauz Khas, New Delhi, India \\ Email: ^rachanagupta07@gmail.com
}

How to cite this paper: Gupta, R. and Mehra, A. (2017) Gap Functions and Error Bounds for Set-Valued Vector Quasi Variational Inequality Problems. Applied Mathematics, 8, 1903-1917.

https://doi.org/10.4236/am.2017.812135

Received: November 17, 2017

Accepted: December 26, 2017

Published: December 29, 2017

Copyright $\odot 2017$ by authors and Scientific Research Publishing Inc. This work is licensed under the Creative Commons Attribution International License (CC BY 4.0).

http://creativecommons.org/licenses/by/4.0/

\begin{abstract}
One of the classical approaches in the analysis of a variational inequality problem is to transform it into an equivalent optimization problem via the notion of gap function. The gap functions are useful tools in deriving the error bounds which provide an estimated distance between a specific point and the exact solution of variational inequality problem. In this paper, we follow a similar approach for set-valued vector quasi variational inequality problems and define the gap functions based on scalarization scheme as well as the one with no scalar parameter. The error bounds results are obtained under fixed point symmetric and locally $\alpha$-Holder assumptions on the set-valued map describing the domain of solution space of a set-valued vector quasi variational inequality problem.
\end{abstract}

\section{Keywords}

Set-Valued Vector Quasi Variational Inequality Problem, Gap Function, Regularized Gap Function, Error Bounds, Fixed Point Symmetric Map, $\alpha$-Holder Map

\section{Introduction}

Let $K: \mathbb{R}^{n} \rightrightarrows \mathbb{R}^{n}$ be a set-valued map such that $K(x)$, for any $x \in \mathbb{R}^{n}$, is a closed convex set in $\mathbb{R}^{n}$. Let $F_{i}: \mathbb{R}^{n} \rightrightarrows \mathbb{R}^{n}, i=1, \cdots, m$ be set-valued maps such that $F_{i}(x), i=1, \cdots, m$ is convex and compact for all $x \in \mathbb{R}^{n}$. Denote by

$$
\begin{aligned}
& \mathbb{R}_{+}^{n}=\left\{y=\left(y_{1}, \cdots, y_{n}\right) \in \mathbb{R}^{n} \mid y_{i} \geq 0, i=1, \cdots, n\right\} \\
& \text { int } \mathbb{R}_{+}^{n}=\left\{y=\left(y_{1}, \cdots, y_{n}\right) \in \mathbb{R}^{n} \mid y_{i}>0, i=1, \cdots, n\right\}
\end{aligned}
$$

The set-valued vector quasi variational inequality ( $S V Q V I)$ problem associated with $F_{i}, i=1, \cdots, m$ and $K$, denoted by $\operatorname{SVQVI}\left(F_{i}, i=1, \cdots, m ; K\right)$, con- 
sists of finding an $x^{*} \in K\left(x^{*}\right)$ such that there exists

$$
f_{i}^{x^{*}} \in F_{i}\left(x^{*}\right), i=1, \cdots, m
$$

and

$$
\left(\left\langle f_{1}^{x^{*}}, y-x^{*}\right\rangle,\left\langle f_{2}^{x^{*}}, y-x^{*}\right\rangle, \cdots,\left\langle f_{m}^{x^{*}}, y-x^{*}\right\rangle\right) \notin-i n t \mathbb{R}_{+}^{m}, \quad \forall y \in K\left(x^{*}\right),
$$

where $\langle\cdot, \cdot\rangle$ denotes the inner product in $\mathbb{R}^{n}$.

Throughout this work, we denote the solution set of $\operatorname{SVQVI}\left(F_{i}, i=1, \cdots, m ; K\right)$ by $\operatorname{sol}\left(\operatorname{SVQVI}\left(F_{i}, i=1, \cdots, m ; K\right)\right)$.

When the set $K(x)$ is a constant set $\bar{K}$ on $\mathbb{R}^{n}$ then $\operatorname{SVQVI}\left(F_{i}, i=1, \cdots, m ; K\right)$ reduces to the following strong vector variational inequality $\operatorname{SVVI}\left(F_{i}, i=1, \cdots, m ; \bar{K}\right)$ in $[1]$.

Find an $x^{*} \in \bar{K}$ such that there exists $f_{i}^{x^{*}} \in F_{i}\left(x^{*}\right), i=1, \cdots, m$ and

$$
\left(\left\langle f_{1}^{x^{*}}, y-x^{*}\right\rangle,\left\langle f_{2}^{x^{*}}, y-x^{*}\right\rangle, \cdots,\left\langle f_{m}^{x^{*}}, y-x^{*}\right\rangle\right) \notin-i n t \mathbb{R}_{+}^{m}, \quad \forall y \in \bar{K} .
$$

Note that if each $F_{i}, i=1, \cdots, m$ is a single-valued map, and $K$ is a constant map $\bar{K}$, then $\operatorname{SVQVI}\left(F_{i}, i=1, \cdots, m ; K\right)$ reduces to the weak Stampacchia vector variational inequality problem $(S V V I)^{w}$ studied in [2].

Quasi variational inequality $(Q V I)$ problems started with a pioneer work of Bensoussan and Lions in 1973. The terminology quasi variational inequality was coined by Bensoussan et al. [3]. A QVI QVI is an extension of a variational inequality ( $V I$ ) [4] in which the underlying set $K$ depends on the solution vector $x$. For further details on $Q V I$ and its applications in various domains, the readers can refer to [5] [6] [7] [8] and the references therein.

In 1980, Giannessi [9] introduced and studied vector variational inequality $(V V I)$ in finite-dimensional Euclidean space. Chen and Cheng [10] studied the $V V I$ in infinite-dimensional spaces and applied it to vector optimization problem. Lee et al. [11] [12], Lin et al. [13], Konnov and Yao [14], and Daniilidis and Hadiisawas [15] studied the generalized $V V I$ and obtained some existence results. Very recently, Charitha et al. [2] presented several scalar-valued gap functions for Stampacchia and Minty-type VVIs. A good source of material on VVI is a research monograph [16]. Motivated by the extension of $V I$ to $V V I$, several researchers initiated the study of $Q V I$ for vector-valued functions, known as vector quasi variational inequalities ( VQVI); see, for instance [11] [12] [13] [14] [15] and the references therein.

In this paper, we first proposed a gap function for $\operatorname{SVQVI}\left(F_{i}, i=1, \cdots, m ; K\right)$ using a scalarization scheme and then developed another scalar-valued gap function for the same problem but without involving any scalar parameter. Under certain monotonicity conditions and fixed point symmetric assumptions, we developed the error bound results for both kinds of gap functions and their regularized counterparts. Further, we relaxed and replaced the fixed point symmetric condition by a locally $\alpha$-Holder condition and obtained the same error bound results. 
We now briefly sketch the contents of the paper. In Section 2, we present a scalarization scheme. In Section 3, we develop the classical gap function and the regularized gap function for $\operatorname{SVQVI}\left(F_{i}, i=1, \cdots, m ; K\right)$ with the help of set-valued scalar quasi variational inequality $(S S Q V I)$. In Section 4, we introduce another scalar gap function and its regularized version for $\operatorname{SVQVI}\left(F_{i}, i=1, \cdots, m ; K\right)$, both free of any scalar parameter. We also develop the error bounds using fixed point symmetric hypothesis on the underlying map $K$. In Section 5, we showed that the same error bounds results can be obtained by relaxing the fixed point symmetric property by the $\alpha$-Holder type hypothesis on $K$.

\section{Scalarization}

In this section, we investigate $\operatorname{SVQVI}\left(F_{i}, i=1, \cdots, m ; K\right)$ via the scalarization approach of Mastroeni [1] and Konnov [17]. We introduce SSQVIfor $\operatorname{SVQVI}\left(F_{i}, i=1, \cdots, m ; K\right)$ and establish an equivalence between them under certain conditions.

Define functions $F_{0}, F, F^{u}: \mathbb{R}^{n} \rightrightarrows \mathbb{R}^{n}$ by following

$$
F_{0}(x)=\operatorname{conv}\left\{F_{i}(x)\right\}_{i=1, \cdots, m}
$$

- $=\left\{z \in \mathbb{R}^{n} \mid z=\sum_{i=1}^{m} \lambda_{i} f_{i}, f_{i} \in F_{i}(x), \lambda_{i} \in \mathbb{R}_{+}, \sum_{i=1}^{m} \lambda_{i}=1\right\}$

- $F(x)=\prod_{i=1}^{m} F_{i}(x)$

- $F^{u}(x)=\bigcup_{i=1}^{m} F_{i}(x)$

Lemma 2.1. Let $F_{i}(x), i=1, \cdots, m$ be nonempty subsets of $\mathbb{R}^{n}$. Then

$$
F^{u}(x) \subseteq F_{0}(x)=\operatorname{conv}\left\{F^{u}(x)\right\}
$$

where conv means convex hull.

Proof. Note that for each $i=1, \cdots, m, \quad F_{i}(x) \subseteq \operatorname{conv}\left\{F_{i}(x)\right\}_{i=1, \cdots, m}$, hence

$$
F^{u}(x) \subseteq F_{0}(x)
$$

Moreover, $F_{0}(x)$ is convex, thus,

$$
\operatorname{conv}\left\{\bigcup_{i=1}^{m} F_{i}(x)\right\}=\operatorname{conv}\left\{F^{u}(x)\right\} \subseteq F_{0}(x)
$$

Conversely, let $x \in F_{0}(x)$. Then, there exist $x_{i} \in F_{i}(x) \subseteq \bigcup_{i=1}^{m} F_{i}(x), i=1, \cdots, m$ and $\lambda_{i} \geq 0, i=1, \cdots, m$ with $\sum_{i=1}^{m} \lambda_{i}=1$, such that $x=\sum_{i=1}^{m} \lambda_{i} x_{i}$, implying $x \in \operatorname{conv}\left\{\bigcup_{i=1}^{m} F_{i}(x)\right\}$. Hence the requisite result follows.

Proposition 2.1. [1] Let $F_{i}(x), i=1, \cdots, m$ be nonempty subsets of $\mathbb{R}^{n}$. For $x \in \mathbb{R}^{n}$, if $F_{i}(x), i=1, \cdots, m$ are compact then, $F(x), F^{u}(x)$ and $F_{0}(x)$ are compact.

The SSQVI associated with set-valued maps $F_{0}$ and $K$, denoted by $\operatorname{SSQVI}\left(F_{0}, K\right)$, consists of finding an $x^{*} \in K\left(x^{*}\right)$ such that there exists 
$f_{0}^{x^{*}} \in F_{0}\left(x^{*}\right)$ and

$$
\left\langle f_{0}^{x^{*}}, y-x^{*}\right\rangle \geq 0, \quad \forall y \in K\left(x^{*}\right)
$$

Throughout this paper, the solution set of $\operatorname{SSQVI}\left(F_{0}, K\right)$ is represented by $\operatorname{sol}\left(\operatorname{SSQVI}\left(F_{0}, K\right)\right)$.

Theorem 2.1. Consider the following

1) $F_{i}, i=1, \cdots, m$ are nonempty, convex and compact valued maps.

2) $K: \mathbb{R}^{n} \rightrightarrows \mathbb{R}^{n}$ is closed, convex valued map.

Then, for each $x \in \mathbb{R}^{n}, \operatorname{sol}\left(\operatorname{SSQVI}\left(F_{0}, K\right)\right)=\operatorname{sol}\left(\operatorname{SVQVI}\left(F_{i}, i=1, \cdots, m ; K\right)\right)$.

Proof. Let $x^{*} \in \operatorname{Sol}\left(\operatorname{SSQVI}\left(F_{0}, K\right)\right)$. Then there exist $f_{0}^{x^{*}} \in F_{0}\left(x^{*}\right)$ such that

$$
\left\langle f_{0}^{x^{*}}, y-x^{*}\right\rangle \geq 0, \quad \forall y \in K\left(x^{*}\right)
$$

By definition of $F_{0}$, there exists $\lambda \in \mathbb{R}_{+}^{m}$, with $\sum_{i=1}^{m} \lambda_{i}=1$ and $f_{i}^{x^{*}} \in F_{i}\left(x^{*}\right), i=1, \cdots, m$, such that

$$
\left\langle\sum_{i=1}^{m} \lambda_{i} f_{i}^{x^{*}}, y-x^{*}\right\rangle \geq 0, \quad \forall y \in K\left(x^{*}\right)
$$

which implies that, for every $y \in K\left(x^{*}\right)$, there exists an index $i_{y}$, such that

$$
\left\langle f_{i_{y}}^{x^{*}}, y-x^{*}\right\rangle \geq 0
$$

It follows that

$$
\left(\left\langle f_{1}^{x^{*}}, y-x^{*}\right\rangle,\left\langle f_{2}^{x^{*}}, y-x^{*}\right\rangle, \cdots,\left\langle f_{m}^{x^{*}}, y-x^{*}\right\rangle\right) \notin-i n t \mathbb{R}_{+}^{m}, \quad \forall y \in K\left(x^{*}\right)
$$

so, $\operatorname{sol}\left(\operatorname{SSQVI}\left(F_{0}, K\right)\right) \subset \operatorname{sol}\left(\operatorname{SVQVI}\left(F_{i}, i=1, \cdots, m ; K\right)\right)$.

Conversely, let $x^{*} \in \operatorname{sol}\left(\operatorname{SVQVI}\left(F_{i}, i=1, \cdots, m ; K\right)\right)$. Hence, $x^{*} \in K\left(x^{*}\right)$, and there exists $f_{i}^{x^{*}} \in F_{i}\left(x^{*}\right), i=1, \cdots, m$, such that

$$
\left(\left\langle f_{1}^{x^{*}}, y-x^{*}\right\rangle,\left\langle f_{2}^{x^{*}}, y-x^{*}\right\rangle, \cdots,\left\langle f_{m}^{x^{*}}, y-x^{*}\right\rangle\right) \notin-i n t \mathbb{R}_{+}^{m}, \quad \forall y \in K\left(x^{*}\right)
$$

thus, for each $y \in K\left(x^{*}\right)$, there exists an index $i_{y}$ such that

$$
\left\langle f_{i_{y}}^{x^{*}}, y-x^{*}\right\rangle \geq 0
$$

Observe that $f_{i_{y}}^{x^{*}} \in F_{0}\left(x^{*}\right)$, hence for each $y \in K\left(x^{*}\right)$, there exist $f_{0}^{*}=f_{i_{y}}^{x^{*}} \in F_{0}\left(x^{*}\right)$ such that

$$
\left\langle f_{0}^{*}, x^{*}-y\right\rangle \leq 0
$$

Consequently,

$$
\sup _{y \in K\left(x^{*}\right)} \min _{f_{0}^{*} \in F_{0}\left(x^{*}\right)}\left\langle f_{0}^{*}, x^{*}-y\right\rangle \leq 0
$$

Under assumption (1) and by Proposition 2.1, $F_{0}\left(x^{*}\right)$ is convex and compact which along with assumption (2) and the minmax theorem, yields

$$
\min _{f_{0}^{*} \in F_{0}\left(x^{*}\right)} \sup _{y \in K\left(x^{*}\right)}\left\langle f_{0}^{*}, x^{*}-y\right\rangle \leq 0
$$


Finally, there exists $f_{0}^{x^{*}} \in F_{0}\left(x^{*}\right)$ such that

$$
\left\langle f_{0}^{x^{*}}, y-x^{*}\right\rangle \geq 0, \quad \forall y \in K\left(x^{*}\right)
$$

completing the requisite result.

\section{Gap Functions by Scalarization}

One of the classical approaches in the analysis of $V I$ and $Q V I$ and its different variants is to transform the inequality into an equivalent constrained or unconstrained optimization problem by means of the notion of gap function, please see, [5] [18] [19] and references cited therein. The gap functions have potential to play an important role in developing iterative algorithms for solving the inequality, analyzing the convergence properties and obtaining useful stopping rules for iterative algorithms. This prompted us to study and analyze different gap functions for $\operatorname{SVQVI}\left(F_{i}, i=1, \cdots, m ; K\right)$.

Definition 3.1. A function $g: \mathbb{R}^{n} \rightarrow \mathbb{R}$ is said to be a gap function for a $\operatorname{SVQVI}\left(F_{i}, i=1, \cdots, m ; K\right)$ on any set $\mathcal{K} \subseteq \mathbb{R}^{n}$ if it satisfies the following properties:

1) $g(x) \geq 0, \forall x \in \mathcal{K}$,

2) $g\left(x^{*}\right)=0, x^{*} \in \mathcal{K} \Leftrightarrow x^{*} \in \operatorname{sol}\left(\operatorname{SVQVI}\left(F_{i}, i=1, \cdots, m ; K\right)\right)$.

\subsection{Classical Gap Function by Scalarization}

Consider the function $g^{F_{0}}: \mathbb{R}^{n} \rightarrow \mathbb{R}$ defined by

$$
g^{F_{0}}(x)=\inf _{f^{x} \in F_{0}(x)} \sup _{y \in K(x)}\left\langle f^{x}, x-y\right\rangle
$$

Theorem 3.1. Consider the following

1) $F_{i}, i=1, \cdots, m$ are nonempty, convex and compact valued maps.

2) $K: \mathbb{R}^{n} \rightrightarrows \mathbb{R}^{n}$ is closed, convex valued map.

Then, $g^{F_{0}}$ defined in (1) is a gap function for $\operatorname{SSQVI}\left(F_{0}, K\right)$ on $\mathcal{K}=\left\{x \in \mathbb{R}^{n} \mid x \in K(x)\right\}$.

Proof. Observe that, for $x \in \mathcal{K}, x \in K(x)$ which implies $g^{F_{0}}(x) \geq 0$.

Next for $x^{*} \in \mathcal{K}, g^{F_{0}}\left(x^{*}\right)=0$ if and only if

$$
\inf _{f^{x^{*}} \in F_{0}\left(x^{*}\right)} \sup _{y \in K\left(x^{*}\right)}\left\langle f^{x^{*}}, x^{*}-y\right\rangle=0
$$

By Proposition 2.1, since $F_{0}\left(x^{*}\right)$ is compact set on $\mathcal{K}$ and $x^{*} \in \mathcal{K}$, there exists $f_{0}^{x^{*}} \in F_{0}\left(x^{*}\right)$ such that

$$
\sup _{y \in K\left(x^{*}\right)}\left\langle f_{0}^{x^{*}}, x^{*}-y\right\rangle=0
$$

therefore, we have

$$
\left\langle f_{0}^{x^{*}}, y-x^{*}\right\rangle \geq 0, \quad \forall y \in K\left(x^{*}\right) .
$$

By invoking Theorem 2.1, $x^{*} \in \operatorname{sol}\left(\operatorname{SVQVI}\left(F_{i}, i=1, \cdots, m ; K\right)\right)$.

The function $g^{F_{0}}$ is not differentiable, in general, an observation that leads 
to consider the regularized gap function.

\subsection{Regularized Gap Function by Scalarization}

For any $\theta>0$, consider the function $g_{\theta}^{F_{0}}: \mathbb{R}^{n} \rightarrow \mathbb{R}$ defined by

$$
g_{\theta}^{F_{0}}(x)=\inf _{f^{x} \in F_{0}(x)} \sup _{y \in K(x)}\left(\left\langle f^{x}, x-y\right\rangle-\frac{1}{2 \theta}\|x-y\|^{2}\right)
$$

If, for $x \in \mathbb{R}^{n}$, each $F_{i}(x), i=1, \cdots, m$ is a compact set and $K(x)$ is a convex set, then by the minimax theorem

$$
g_{\theta}^{F_{0}}(x)=\sup _{y \in K(x)} \inf _{f^{x} \in F_{0}(x)}\left(\left\langle f^{x}, x-y\right\rangle-\frac{1}{2 \theta}\|x-y\|^{2}\right)=\sup _{y \in K(x)} h(x, y)
$$

where $h(x, y)=\inf _{f^{x} \in F_{0}(x)}\left(\left\langle f^{x}, x-y\right\rangle-\frac{1}{2 \theta}\|x-y\|^{2}\right)$.

Since $h(x, \cdot)$ is a strongly concave function in $y$ so has unique maxima over closed convex set $K(x)$, then follow from [20] (Chapter 4, Theorem 1.7), $g_{\theta}^{F_{0}}$ is differentiable on $\mathbb{R}^{n}$.

Note that if $F_{0}(x)$ is a singleton then this gap function reduces to the regularized gap function for QVI proposed by Taji [19].

Theorem 3.2. Consider the following

1) $F_{i}, i=1, \cdots, m$ are nonempty, convex and compact valued maps.

2) $K: \mathbb{R}^{n} \rightrightarrows \mathbb{R}^{n}$ is closed, convex valued map.

Then, $g_{\theta}^{F_{0}}$ is a gap function for $\operatorname{SVQVI}\left(F_{i}, i=1, \cdots, m ; K\right)$ over $\mathcal{K}$.

Proof. Clearly, for $x \in \mathcal{K}, \quad g_{\theta}^{F_{0}}(x) \geq 0$.

Let $g_{\theta}^{F_{0}}\left(x^{*}\right)=0$ and $x^{*} \in \mathcal{K}$. Then,

$$
\inf _{f^{*^{*}} \in F_{0}\left(x^{*}\right)} \sup _{y \in K\left(x^{*}\right)}\left(\left\langle f^{x^{*}}, x^{*}-y\right\rangle-\frac{1}{2 \theta}\left\|x^{*}-y\right\|^{2}\right)=0
$$

Under assumption (1) and by Proposition 2.1, there exists $f_{0}^{x^{*}} \in F_{0}\left(x^{*}\right)$ such that

$$
\sup _{y \in K\left(x^{*}\right)}\left(\left\langle f_{0}^{x^{*}}, x^{*}-y\right\rangle-\frac{1}{2 \theta}\left\|x^{*}-y\right\|^{2}\right)=0,
$$

which implies

$$
\left\langle f_{0}^{x^{*}}, x^{*}-y\right\rangle-\frac{1}{2 \theta}\left\|x^{*}-y\right\|^{2} \leq 0, \quad \forall y \in K\left(x^{*}\right)
$$

Take an arbitrary point $z \in K\left(x^{*}\right)$, and define a sequence of vectors $y_{k}$ as

$$
y_{k}=x^{*}+\frac{1}{k}\left(z-x^{*}\right), \quad k \in \mathbb{N}
$$

$K\left(x^{*}\right)$ being convex, so $y_{k} \in K\left(x^{*}\right), k \in \mathbb{N}$, therefore

$$
\left\langle f_{0}^{x^{*}}, x^{*}-y_{k}\right\rangle \leq \frac{1}{2 \theta}\left\|x^{*}-y_{k}\right\|^{2}
$$

which when $k \rightarrow \infty$ yields

$$
\left\langle f_{0}^{x^{*}}, z-x^{*}\right\rangle \geq 0
$$


Hence $x^{*} \in \operatorname{Sol}\left(\operatorname{SSQVI}\left(F_{0}, K\right)\right)$, which implies that $x^{*} \in \operatorname{sol}\left(\operatorname{SVQVI}\left(F_{i}, i=1, \cdots, m ; K\right)\right)$ also.

Conversely, let $x^{*} \in \operatorname{sol}\left(\operatorname{SVQVI}\left(F_{i}, i=1, \cdots, m ; K\right)\right)$. Then, by Theorem 2.1, $x^{*} \in \operatorname{sol}\left(\operatorname{SSQVI}\left(F_{0}, K\right)\right)$. Hence $x^{*} \in K\left(x^{*}\right)$ and there exists $f_{0}^{x^{*}} \in F_{0}\left(x^{*}\right)$ such that

$$
\sup _{y \in K\left(x^{*}\right)}\left\langle f_{0}^{x^{*}}, x^{*}-y\right\rangle \leq 0
$$

therefore

$$
g_{\theta}^{F_{0}}\left(x^{*}\right)=\inf _{f^{x^{*}} \in F_{0}\left(x^{*}\right)} \sup _{y \in K\left(x^{*}\right)}\left(\left\langle f^{x^{*}}, x^{*}-y\right\rangle-\frac{1}{2 \theta}\left\|x^{*}-y\right\|^{2}\right) \leq 0
$$

But $g_{\theta}^{F_{0}}\left(x^{*}\right) \geq 0$, which gives $g_{\theta}^{F_{0}}\left(x^{*}\right)=0$.

\section{Another Scalar Gap Functions for SVQVI}

In previous section, we used the scalarization parameter $\lambda$ in constructing $\operatorname{SSQVI}\left(F_{0}, K\right)$ and then studied the gap function for $\operatorname{SVQVI}\left(F_{i}, i=1, \cdots, m ; K\right)$. It is interesting to ask whether one can develop a gap function for $\operatorname{SVQVI}\left(F_{i}, i=1, \cdots, m ; K\right)$ without taking help of $\operatorname{SSQVI}\left(F_{0}, K\right)$. We make an attempt to construct such a gap function in the discussion to follow. But first a notation.

Let $x, y \in K(x)$ and let $f^{x}=\left(f_{1}^{x}, \cdots, f_{m}^{x}\right) \in F(x)$. Then, $f_{i}^{x} \in F_{i}(x), i=1, \cdots, m$ and denote

$$
\left\langle f^{x}, y-x\right\rangle=\left(\left\langle f_{1}^{x}, y-x\right\rangle,\left\langle f_{2}^{x}, y-x\right\rangle, \ldots,\left\langle f_{m}^{x}, y-x\right\rangle\right),
$$

i.e., $\left\langle f_{i}^{x}, y-x\right\rangle$ is the $i^{\text {th }}$ component of the vector $\left\langle f^{x}, y-x\right\rangle, i=1, \cdots, m$.

\subsection{Classical Gap Function}

Define a function $g: \mathbb{R}^{n} \rightarrow \mathbb{R}$ such that

$$
g(x)=\inf _{f^{x} \in F(x)} \sup _{y \in K(x)} \min _{1 \leq i \leq m}\left\langle f_{i}^{x}, x-y\right\rangle
$$

Theorem 4.1. Consider the following

1) $F_{i}, i=1, \cdots, m$ are nonempty, convex and compact valued.

2) $K: \mathbb{R}^{n} \rightrightarrows \mathbb{R}^{n}$ is closed, convex valued map.

Then, $g$ defined in (2) is a gap function for $\operatorname{SVQVI}\left(F_{i}, i=1, \cdots, m ; K\right)$ on $\mathcal{K}=\left\{x \in \mathbb{R}^{n} \mid x \in K(x)\right\}$.

Proof. Since $x \in \mathcal{K}$, so $x \in K(x)$ which implies $g(x) \geq 0$.

Consider $x^{*} \in \mathcal{K}$. We observe that $g\left(x^{*}\right)=0$ if and only if there exists $f^{x^{*}} \in F\left(x^{*}\right)$ such that

$$
\sup _{y \in K\left(x^{*}\right)} \min _{1 \leq i \leq m}\left\langle f_{i}^{x^{*}}, x^{*}-y\right\rangle=0
$$

that is,

$$
\min _{1 \leq i \leq m}\left\langle f_{i}^{x^{*}}, y-x^{*}\right\rangle \geq 0, \quad \forall y \in K\left(x^{*}\right)
$$


Equivalently,

$$
\left\langle f^{x^{*}}, y-x^{*}\right\rangle \notin-i n t \mathbb{R}_{+}^{m}, \quad \forall y \in K\left(x^{*}\right)
$$

Hence, $x^{*} \in \operatorname{sol}\left(\operatorname{SVQVI}\left(F_{i}, i=1, \cdots, m ; K\right)\right)$.

Proposition 4.1. For each $x \in \mathbb{R}^{n}, g^{F_{0}}(x) \geq g(x)$.

Proof. Let $x \in \mathbb{R}^{n}$ and $f_{0}^{x} \in F_{0}(x)$. Then there exist $f_{i}^{x} \in F_{i}(x)$ or equivalently, $f^{x}=\left(f_{1}^{x}, \cdots, f_{m}^{x}\right) \in F(x) \quad$ and $\quad \lambda_{i} \geq 0, i=1, \cdots, m$ with $\sum_{i=1}^{m} \lambda_{i}=1$ such that $f_{0}^{x}=\sum_{i=1}^{m} \lambda_{i} f_{i}$. For any $y \in K(x)$,

$$
\min _{1 \leq i \leq m}\left\langle f_{i}^{x}, x-y\right\rangle \leq\left\langle\sum_{i=1}^{m} \lambda_{i} f_{i}^{x}, x-y\right\rangle=\left\langle f_{0}^{x}, x-y\right\rangle
$$

It follows that

$$
g(x)=\inf _{f^{x} \in F(x)} \sup _{y \in K(x)} \min _{1<i \leq m}\left\langle f_{i}^{x}, x-y\right\rangle \leq \inf _{f_{0}^{x} \in F_{0}(x)} \sup _{y \in K(x)}\left\langle f_{0}^{x}, x-y\right\rangle=g^{F_{0}}(x)
$$

We now attend to our prime aim that to develop the error bounds for $\operatorname{SVQVI}\left(F_{i}, i=1, \cdots, m ; K\right)$. We shall be needing the following concepts.

Definition 4.1. [1] A set-valued map $F: \mathbb{R}^{n} \rightrightarrows \mathbb{R}^{n}$ is said to be strongly monotone with modulus $\mu>0$ on $\mathbb{R}^{n}$ if, for any $x, y \in \mathbb{R}^{n}$,

$$
\left\langle f^{x}-f^{y}, x-y\right\rangle \geq \mu\|x-y\|^{2}, \quad \forall f^{x} \in F(x), f^{y} \in F(y)
$$

$F$ is said to be monotone if the above inequality holds with $\mu=0 . F$ is said to be strictly monotone if it is monotone and the strict relation in the above inequality holds when $x \neq y$.

Remark 4.1. Let $F_{1}, F_{2}: \mathbb{R}^{n} \rightrightarrows \mathbb{R}^{n} \quad$ be two set-valued maps with $F_{1}(x) \subseteq F_{2}(x)$ for any $x \in \mathbb{R}^{n}$. Note that, if $F_{2}$ is strongly monotone with modulus $\mu>0$ (respectively, monotone and strictly monotone) on $\mathbb{R}^{n}$ then, $F_{1}$ is also strongly monotone with modulus $\mu>0$ (respectively, monotone and strictly monotone) on $\mathbb{R}^{n}$. Consequently, recall if $F^{u}$ is strongly monotone with modulus $\mu>0$ (respectively, monotone and strictly monotone) on $\mathbb{R}^{n}$ then, each $F_{i}, i=1, \cdots, m$ is strongly monotone with modulus $\mu>0$ (respectively, monotone and strictly monotone) on $\mathbb{R}^{n}$.

Remark 4.2. Note that if $F^{u}$ is strongly monotone with modulus $\mu$ on any set $S \subseteq \mathbb{R}^{n}$ then each $F_{i}$ is strongly monotone with modulus $\mu$ on $S$ [1]. However, the converse, in general, may not hold. For instance, consider two maps $F_{1}, F_{2}: \mathbb{R} \rightrightarrows \mathbb{R} \quad$ as $F_{1}(x)=\{x\}$ and $F_{2}(x)=\{3 x\}$. Then, $F_{1}, F_{2}$ are strongly monotone on $\mathbb{R}$ with modulus 1 and 3 respectively. But for $x=1$, $y=2 ; 3 \in F^{u}(1), 2 \in F^{u}(2)$, we have, $\langle 3-2,1-2\rangle=-1$, which means $F^{u}$ is not strongly monotone (not even monotone) map on $\mathbb{R}$.

Definition 4.2. [5] A set-valued map $K: \mathbb{R}^{n} \rightrightarrows \mathbb{R}^{n}$ is said to be fixed point symmetric if for all $x \in \mathcal{K}=\left\{x \in \mathbb{R}^{n} \mid x \in K(x)\right\}$, we have,

$$
\text { if } y \in K(x) \text { then } x \in K(y)
$$

The following result provides an error bound in terms of scalar gap function (without scalarize parameter) under strong monotonicity of $F^{u}$ map and fixed pint symmetric $K$ map. 
Theorem 4.2. Let $x^{*} \in \operatorname{sol}\left(\operatorname{SVQVI}\left(F_{i}, i=1, \cdots, m ; K\right)\right)$. Suppose the following hold

1) $F_{i}, i=1, \cdots, m$ are nonempty, convex, compact valued.

2) $K$ is closed, convex valued and fixed point symmetric map.

3) $F^{u}$ is strongly monotone with modulus $\mu>0$ on $\mathcal{K}$.

Then, for $x \in K\left(x^{*}\right)$, we have

$$
\left\|x-x^{*}\right\| \leq \sqrt{\frac{g(x)}{\mu}}
$$

Proof. Since $x^{*} \in \operatorname{sol}\left(\operatorname{SVQVI}\left(F_{i}, i=1, \cdots, m ; K\right)\right)$, there exists $f_{i}^{x^{*}} \in F_{i}\left(x^{*}\right), i=1, \cdots, m$ such that

$$
\left(\left\langle f_{1}^{x^{*}}, y-x^{*}\right\rangle,\left\langle f_{2}^{x^{*}}, y-x^{*}\right\rangle, \cdots,\left\langle f_{m}^{x^{*}}, y-x^{*}\right\rangle\right) \notin-i n t \mathbb{R}_{+}^{m}, \quad \forall y \in K\left(x^{*}\right)
$$

For $y=x$, we have

$$
\left(\left\langle f_{1}^{x^{*}}, x-x^{*}\right\rangle,\left\langle f_{2}^{x^{*}}, x-x^{*}\right\rangle, \cdots,\left\langle f_{m}^{x^{*}}, x-x^{*}\right\rangle\right) \notin-i n t \mathbb{R}_{+}^{m}
$$

Therefore, there exists an index $i_{x}$ such that $f_{i_{x}}^{x^{*}} \in F_{i_{x}}\left(x^{*}\right) \subseteq F^{u}\left(x^{*}\right)$ and

$$
\left\langle f_{i_{x}}^{x^{*}}, x-x^{*}\right\rangle \geq 0
$$

Now, from the definition of $g(x)$ and by Proposition 2.1, there exists $f^{x} \in F(x), f^{x}=\left(f_{1}^{x}, \cdots, f_{m}^{x}\right)$ such that

$$
g(x)=\sup _{y \in K(x)} \min _{1 \leq i \leq m}\left\langle f_{i}^{x}, x-y\right\rangle
$$

which gives

$$
g(x) \geq \min _{1 \leq i \leq m}\left\langle f_{i}^{x}, x-y\right\rangle, \quad \forall y \in K(x)
$$

Since $x \in K\left(x^{*}\right)$, by fixed point symmetric property of $K, x^{*} \in K(x)$, thus taking $y=x^{*}$ in above inequality, we have

$$
g(x) \geq \min _{1 \leq i \leq m}\left\langle f_{i}^{x}, x-x^{*}\right\rangle
$$

For $f_{i}^{x} \in F_{i}(x) \subseteq F^{u}(x), i=1, \cdots, m$, by strongly monotonicity of $F^{u}$ and (4), we get

$$
g(x) \geq \min _{1 \leq i \leq m}\left\langle f_{i}^{x}-f_{i_{x}}^{x^{*}}, x-x^{*}\right\rangle+\left\langle f_{i_{x}}^{x^{*}}, x-x^{*}\right\rangle \geq \mu\left\|x-x^{*}\right\|^{2}
$$

Hence, for any $x \in K\left(x^{*}\right)$,

$$
\left\|x-x^{*}\right\| \leq \sqrt{\frac{g(x)}{\mu}}
$$

Remark 4.3. We observed that the strong monotonicity of $F^{u}$ (that is, assumption (3)) is used only to obtain relation (6). A careful examination reveals that even the following condition can help us to achieve the same error bound for $\operatorname{SVQVI}\left(F_{i}, i=1, \cdots, m ; K\right)$ :

For any $x \in K\left(x^{*}\right)$ and for any $f^{x}=\left(f_{1}^{x}, \cdots, f_{m}^{x}\right) \in F(x)$, there exists an index $j \in\{1, \cdots, m\}$, and $f_{j}^{x^{*}} \in F_{j}\left(x^{*}\right)$ satisfying (4) and 


$$
\min _{1 \leq i \leq m}\left\langle f_{i}^{x}-f_{j}^{x^{*}}, x-x^{*}\right\rangle \geq \mu\left\|x-x^{*}\right\|^{2}
$$

Hence the error bound given in (3) is valid for $\operatorname{SVQVI}\left(F_{i}, i=1, \cdots, m ; K\right)$ because under assumption (3) of Theorem 4.2, the set-valued maps $F_{i}, i=1, \cdots, m$ always satisfy (7).

In particular, if $K$ is a constant map $\bar{K}$ and each $F_{i}$ is a single-valued map, then (7) states that for any $x \in \bar{K}$, there exists an index $j$ such that

$$
\min _{1<i \leq m}\left\langle F_{i}(x)-F_{j}\left(x^{*}\right), x-x^{*}\right\rangle \geq \mu\left\|x-x^{*}\right\|^{2}
$$

For instant, take $F_{1}, F_{2}: \mathbb{R} \rightrightarrows \mathbb{R}$ given as $F_{1}(x)=\{2 x\}$ and $F_{2}(x)=\left\{x-\frac{1}{2}\right\}$ and $\bar{K}=[-1,+1]$. For this, $\operatorname{sol}\left(\operatorname{SVVI}\left(F_{1}, F_{2} ; K\right)\right)=\left[0, \frac{1}{2}\right]$. In this case $F^{u}$ is not strongly monotone that means assumption (3) of Theorem 4.2 fails but the error bound Formula (3) remains valid because $F_{1}, F_{2}$ satisfy (8).

In light of Proposition 4.1, the following is immediate.

Corollary 4.2.1. Let $x^{*} \in \operatorname{Sol}\left(\operatorname{SVQVI}\left(F_{i}, i=1, \cdots, m ; K\right)\right)$. Suppose the following hold

1) $F_{i}, i=1, \cdots, m$ are nonempty, convex, compact valued.

2) $K$ is closed, convex valued and fixed point symmetric map.

3) $F^{u}$ is strongly monotone with modulus $\mu>0$ on $\mathbb{R}^{n}$.

Then, for $x \in K\left(x^{*}\right)$,

$$
\left\|x-x^{*}\right\| \leq \sqrt{\frac{g^{F_{0}}(x)}{\mu}}
$$

Similar to $g^{F_{0}}$, the gap function $g$ is not differentiable leading to define the regularized gap function for $\operatorname{SVQVI}\left(F_{i}, i=1, \cdots, m ; K\right)$.

\subsection{Regularized Gap Function}

For $\theta>0$, define a function $g_{\theta}: \mathbb{R}^{n} \rightarrow \mathbb{R}$ as

$$
g_{\theta}(x)=\inf _{f^{x} \in F(x)} \sup _{y \in K(x)} \min _{1 \leq i \leq m}\left(\left\langle f_{i}^{x}, x-y\right\rangle-\frac{1}{2 \theta}\|x-y\|^{2}\right)
$$

For each $x$, define the function

$$
\varphi(x, y)=\min _{1 \leq i \leq m}\left(\left\langle f_{i}^{x}, x-y\right\rangle-\frac{1}{2 \theta}\|x-y\|^{2}\right)
$$

Here, $\varphi(x,$.$) is a strongly concave function of y$. When $K(x)$ is a closed convex set for any $x \in \mathbb{R}^{n}$ then, $\varphi(x,$.$) attains maximum at a unique point in$ $K(x)$. If $F(x)$ is a compact set in $\mathbb{R}^{m}$ then, it follow from [20] (Chapter 4, Theorem 1.7), $g_{\theta}$ is differentiable.

Theorem 4.3. Consider the following

1) $F_{i}, i=1, \cdots, m$ are nonempty, convex and compact valued.

2) $K: \mathbb{R}^{n} \rightrightarrows \mathbb{R}^{n}$ is closed, convex valued map.

Then, $g_{\theta}$ defined in (9) is a gap function for $\operatorname{SVQVI}\left(F_{i}, i=1, \cdots, m ; K\right)$ over the set $\mathcal{K}$. 
Proof. Since $x \in \mathcal{K}$, so $x \in K(x)$ which implies $g_{\theta}(x) \geq 0$.

Let $x^{*} \in \mathcal{K}$. We observe that $g_{\theta}\left(x^{*}\right)=0$ if there exists $f^{x^{*}} \in F\left(x^{*}\right)$ such that

$$
\sup _{y \in K\left(x^{*}\right)} \min _{1<i \leq m}\left\{\left\langle f_{i}^{x^{*}}, x^{*}-y\right\rangle-\frac{1}{2 \theta}\|x-y\|^{2}\right\}=0
$$

By similar arguments given in Theorem 3.2, we can work out that

$$
\min _{1 \leq i \leq m}\left\langle f_{i}^{x^{*}}, x^{*}-y\right\rangle \leq 0, \quad \forall y \in K\left(x^{*}\right)
$$

which is equivalent to

$$
\left\langle f^{x^{*}}, y-x^{*}\right\rangle \notin-i n t \mathbb{R}_{+}^{m}, \quad \forall y \in K\left(x^{*}\right)
$$

that is, $x^{*} \in \operatorname{sol}\left(\operatorname{SVQVI}\left(F_{i}, i=1, \cdots, m ; K\right)\right)$.

For the converse part, let $x^{*} \in \operatorname{sol}\left(\operatorname{SVQVI}\left(F_{i}, i=1, \cdots, m ; K\right)\right)$. Then $x^{*} \in K\left(x^{*}\right)$ and there exists $f_{i}^{x^{*}} \in F_{i}\left(x^{*}\right), i=1, \cdots, m$ such that

$$
\left(\left\langle f_{1}^{x^{*}}, y-x^{*}\right\rangle,\left\langle f_{2}^{x^{*}}, y-x^{*}\right\rangle, \cdots,\left\langle f_{m}^{x^{*}}, y-x^{*}\right\rangle\right) \notin-i n t \mathbb{R}_{+}^{m}, \quad \forall y \in K\left(x^{*}\right)
$$

Hence for any arbitrary but fixed $z \in K\left(x^{*}\right)$, there exists an index $i_{z}$, depending on $z$, and there exists $f_{i_{z}}^{x^{*}} \in F_{i_{z}}\left(x^{*}\right)$, such that

$$
\left\langle f_{i_{z}}^{x^{*}}, x^{*}-z\right\rangle \leq 0
$$

In other words,

$$
\min _{1 \leq i \leq m}\left\{\left\langle f_{i}^{x^{*}}, x^{*}-y\right\rangle-\frac{1}{2 \theta}\left\|x^{*}-y\right\|^{2}\right\} \leq 0, \quad \forall y \in K\left(x^{*}\right)
$$

which implies

$$
g_{\theta}\left(x^{*}\right)=\inf _{f^{x^{*}} \in F\left(x^{*}\right)} \sup _{y \in K\left(x^{*}\right)} \min _{1<i \leq m}\left(\left\langle f_{i}^{x^{*}}, x^{*}-y\right\rangle-\frac{1}{2 \theta}\left\|x^{*}-y\right\|^{2}\right) \leq 0
$$

We conclude that $g_{\theta}\left(x^{*}\right)=0$, and hence the result follows.

Theorem 4.4. Let $x^{*} \in \operatorname{Sol}\left(\operatorname{SVQVI}\left(F_{i}, i=1, \cdots, m ; K\right)\right)$. Suppose the following hold

1) $F_{i}, i=1, \cdots, m$ are nonempty, convex, compact valued.

2) $K$ is closed, convex valued and fixed point symmetric map.

3) $F^{u}$ is strongly monotone with modulus $\mu>0$ on $\mathcal{K}$.

Then, for $\theta>\frac{1}{2 \mu}$ and for any $x \in K\left(x^{*}\right)$,

$$
\left\|x-x^{*}\right\| \leq \sqrt{\frac{g_{\theta}(x)}{\left(\mu-\frac{1}{2 \theta}\right)}}
$$

Proof. Since $x^{*} \in \operatorname{sol}\left(\operatorname{SVQVI}\left(F_{i}, i=1, \cdots, m ; K\right)\right)$, there exists $f_{i}^{x^{*}} \in F_{i}\left(x^{*}\right), i=1, \cdots, m$ such that

$$
\left(\left\langle f_{1}^{x^{*}}, y-x^{*}\right\rangle,\left\langle f_{2}^{x^{*}}, y-x^{*}\right\rangle, \cdots,\left\langle f_{m}^{x^{*}}, y-x^{*}\right\rangle\right) \notin-i n t \mathbb{R}_{+}^{m}, \quad \forall y \in K\left(x^{*}\right)
$$


Taking $y=x \in K\left(x^{*}\right)$, we have

$$
\left(\left\langle f_{1}^{x^{*}}, x-x^{*}\right\rangle,\left\langle f_{2}^{x^{*}}, x-x^{*}\right\rangle, \cdots,\left\langle f_{m}^{x^{*}}, x-x^{*}\right\rangle\right) \notin-i n t \mathbb{R}_{+}^{m}
$$

There exists an index $i_{x}$ such that $f_{i_{x}}^{x^{*}} \in F_{i_{x}}\left(x^{*}\right) \subseteq F^{u}\left(x^{*}\right)$, and

$$
\left\langle f_{i_{x}}^{x^{*}}, x-x^{*}\right\rangle \geq 0
$$

Proceeding along the lines of Theorem 4.2, we can easily obtain, for $x \in K\left(x^{*}\right)$,

$$
\begin{aligned}
g_{\theta}(x) & \geq \min _{1 \leq i \leq m}\left\langle f_{i}^{x}-f_{i_{x}}^{x^{*}}, x-x^{*}\right\rangle+\left\langle f_{i_{x}}^{x^{*}}, x-x^{*}\right\rangle-\frac{1}{2 \theta}\left\|x-x^{*}\right\|^{2} \\
& \geq\left(\mu-\frac{1}{2 \theta}\right)\left\|x-x^{*}\right\|^{2}
\end{aligned}
$$

where the last inequality follows from strongly monotonicity of $F^{u}$ and (10), yielding the requisite result.

\section{Substitution of "Fixed Point Symmetric Assumption"}

Aussel [5] obtained the error bounds for a $S S Q V I$ by replacing "fixed point symmetric" property on $K$ by the Holder's type hypothesis which motivated us to see if the Holder's type hypothesis on $K$ works for $\operatorname{SVQVI}\left(F_{i}, i=1, \cdots, m ; K\right)$ too.

Definition 5.1. [5] A set-valued map $K$ is said to be locally $\alpha$-Holder $(\alpha>0)$ at a point $x^{*} \in \mathbb{R}^{n}$ if there exists $\delta>0$ and $L>0$ such that for all $x \in B\left(x^{*}, \delta\right)$

$$
K\left(x^{*}\right) \cap B\left(x^{*}, \delta\right) \subset K(x)+B\left(0, L\left\|x-x^{*}\right\|^{\alpha}\right)
$$

where $B$ represents a ball in $\mathbb{R}^{n}$.

Remark 5.1. If $K: \mathbb{R}^{n} \rightrightarrows \mathbb{R}^{n}$ is a fixed point symmetric map over any set $S \subseteq \mathbb{R}^{n}$ then $K$ will also be locally $\alpha$-Holder $(\alpha \geq 1, L \geq 1)$ at any point $x \in S$. However, the converse, in general, may not hold. For instance, consider Proposition 3.6 in [5], where the constraints map $K$ is defined, for any $x \in \mathbb{R}^{n}$, by

$$
K(x)=\left\{y \in \mathbb{R}^{n} \mid \phi(y) \leq \psi(x)\right\}
$$

where $\phi: \mathbb{R}^{n} \rightarrow \mathbb{R}$ is a continuously differentiable function and $\psi: \mathbb{R}^{n} \rightarrow \mathbb{R}$ is an $\alpha$-Holder continuous on $\mathbb{R}^{n}$. Let $x \in S$ be such that $\nabla \phi(x) \neq 0$. Then for some constant $\gamma$ (see Proposition 3.6 in [5]), the constraint map $K$ is locally $\gamma$-Holder at $x \in S$. Note that $K$ is not necessary fixed point symmetric over $S$.

Recall the map $F^{u}: \mathbb{R}^{n} \rightrightarrows \mathbb{R}^{n}$. For if $F^{u}$ is a compact valued map then define

$$
M=\sup \left\{\|f\|: f \in F^{u}(x), \forall x \in \bar{B}\left(x^{*}, 1\right)\right\}
$$

where $\bar{B}\left(x^{*}, 1\right)$ indicates the closed unit ball in $\mathbb{R}^{n}$ centered at $x^{*}$.

Theorem 5.1. Let $x^{*} \in \operatorname{sol}\left(\operatorname{SVQVI}\left(F_{i}, i=1, \cdots, m ; K\right)\right)$. Suppose the following hold 
1) $F_{i}, i=1, \cdots, m$ are nonempty, convex, compact valued.

2) $K$ is closed, convex valued and locally $\alpha$-Holder with $\alpha>2$ at $x^{*}$ and $\delta \in(0,1)$.

3) $F^{u}$ is strongly monotone with modulus $\mu>M L \delta^{\alpha-2}>0$.

Then, for any $\theta>\frac{(L+1)^{2}}{2\left(\mu-M L \delta^{\alpha-2}\right)}$, and for any

$$
x \in B\left(x^{*}, \delta\right) \cap K\left(x^{*}\right),\left\|x-x^{*}\right\| \leq \sqrt{\frac{g_{\theta}(x)}{\rho_{\delta}}}
$$

where $\rho_{\delta}=\left(\mu-M L \delta^{\alpha-2}-\frac{(L+1)^{2}}{2 \theta}\right)$.

Proof. Since $x^{*} \in \operatorname{sol}\left(\operatorname{SVQVI}\left(F_{i}, i=1, \cdots, m ; K\right)\right)$, there exists $f_{i}^{x^{*}} \in F_{i}\left(x^{*}\right), i=1, \cdots, m$ such that

$$
\left(\left\langle f_{1}^{x^{*}}, y-x^{*}\right\rangle,\left\langle f_{2}^{x^{*}}, y-x^{*}\right\rangle, \cdots,\left\langle f_{m}^{x^{*}}, y-x^{*}\right\rangle\right) \notin-i n t \mathbb{R}_{+}^{m}, \quad \forall y \in K\left(x^{*}\right)
$$

Taking $y=x$ in above relation

$$
\left(\left\langle f_{1}^{x^{*}}, x-x^{*}\right\rangle,\left\langle f_{2}^{x^{*}}, x-x^{*}\right\rangle, \cdots,\left\langle f_{m}^{x^{*}}, x-x^{*}\right\rangle\right) \notin-i n t \mathbb{R}_{+}^{m}
$$

Hence, there exists an index $i_{x}$ and $f_{i_{x}}^{x^{*}} \in F_{i_{x}}\left(x^{*}\right)$ such that

$$
\left\langle f_{i_{x}}^{x^{*}}, x-x^{*}\right\rangle \geq 0
$$

Also,

$$
g_{\theta}(x)=\inf _{f^{x} \in F(x)} \sup _{y \in K(x)} \min _{1 \leq i \leq m}\left(\left\langle f_{i}^{x}, x-y\right\rangle-\frac{1}{2 \theta}\|y-x\|^{2}\right)
$$

Using Proposition 2.1, there exists $f^{x} \in F(x)$ such that

$$
g_{\theta}(x) \geq \min _{1 \leq i \leq m}\left(\left\langle f_{i}^{x}, x-y\right\rangle-\frac{1}{2 \theta}\|y-x\|^{2}\right), \quad \forall y \in K(x)
$$

For any $y \in K(x)$, there exists an index $i_{y}$ and $f_{i_{y}}^{x} \in F_{i_{y}}(x)$ such that

$$
g_{\theta}(x) \geq\left\langle f_{i_{y}}^{x}, x-y\right\rangle-\frac{1}{2 \theta}\|y-x\|^{2}
$$

Consequently,

$$
\begin{aligned}
g_{\theta}(x) & \geq\left\langle f_{i_{y}}^{x}, x-y\right\rangle-\frac{1}{2 \theta}\|y-x\|^{2} \\
& =\left\langle f_{i_{y}}^{x}, x-x^{*}\right\rangle+\left\langle f_{i_{y}}^{x}, x^{*}-y\right\rangle-\frac{1}{2 \theta}\|y-x\|^{2} \\
& =\left\langle f_{i_{y}}^{x}-f_{i_{x}}^{x^{*}}, x-x^{*}\right\rangle+\left\langle f_{i_{x} x^{*}}, x-x^{*}\right\rangle+\left\langle f_{i_{y}}^{x}, x^{*}-y\right\rangle-\frac{1}{2 \theta}\|y-x\|^{2} \\
& \geq \mu\left\|x-x^{*}\right\|^{2}+\left\langle f_{i_{y}}^{x}, x^{*}-y\right\rangle-\frac{1}{2 \theta}\left(\left\|y-x^{*}\right\|+\left\|x^{*}-x\right\|\right)^{2},
\end{aligned}
$$

where the last inequality is due to assumption (3), (11) and triangular inequality of $\|\cdot\|$.

Since $K$ is locally $\alpha$-Holder at $x^{*}$, for all $x \in B\left(x^{*}, \delta\right) \cap K\left(x^{*}\right)$, we have

$$
\left\|x^{*}-y\right\| \leq L\left\|x^{*}-x\right\|^{\alpha}, \quad \forall y \in K(x)
$$


Taking into account that $\left\|x-x^{*}\right\|<1$, inequality (12), we have, for $x \in B\left(x^{*}, \delta\right) \cap K\left(x^{*}\right)$,

$$
\begin{aligned}
g_{\theta}(x) & \geq \mu\left\|x-x^{*}\right\|^{2}-M\left\|x^{*}-y\right\|-\frac{1}{2 \theta}\left(\left\|y-x^{*}\right\|+\left\|x-x^{*}\right\|\right)^{2} \\
& \geq \mu\left\|x-x^{*}\right\|^{2}-M L\left\|x-x^{*}\right\|^{\alpha}-\frac{1}{2 \theta}\left(L\left\|x-x^{*}\right\|^{\alpha}+\left\|x-x^{*}\right\|\right)^{2} \\
& =\mu\left\|x-x^{*}\right\|^{2}-M L\left\|x-x^{*}\right\|^{\alpha}-\frac{1}{2 \theta}\left(L^{2}\left\|x-x^{*}\right\|^{2 \alpha}+2 L\left\|x-x^{*}\right\|^{\alpha+1}+\left\|x-x^{*}\right\|^{2}\right) \\
& \geq\left(\mu-M L\left\|x-x^{*}\right\|^{\alpha-2}-\frac{1}{2 \theta}-\frac{L}{\theta}-\frac{L^{2}}{2 \theta}\right)\left\|x-x^{*}\right\|^{2} \\
& \geq\left(\mu-M L \delta^{\alpha-2}-\frac{(L+1)^{2}}{2 \theta}\right)\left\|x-x^{*}\right\|^{2}=\rho_{\delta}\left\|x-x^{*}\right\|^{2},
\end{aligned}
$$

where $\rho_{\delta}=\left(\mu-M L \delta^{\alpha-2}-\frac{(L+1)^{2}}{2 \theta}\right)$.

Then, for all $x \in B\left(x^{*}, \delta\right) \cap K\left(x^{*}\right)$, if $x \neq x^{*}$ we have $g_{\theta}(x)>0$ because $\rho_{\delta}>0$, thus proving that $x^{*}$ is the unique solution of $\operatorname{SVQVI}\left(F_{i}, i=1, \cdots, m ; K\right)$ over $B\left(x^{*}, \delta\right) \cap K\left(x^{*}\right)$.

\section{References}

[1] Li, J. and Mastroeni, G. (2010) Vector Variational Inequalities Involving Set-Valued Mappings via Scalarization with Applications to Error Bounds for Gap Functions. Journal of Optimization Theory and Applications, 145, 355-372. https://doi.org/10.1007/s10957-009-9625-1

[2] Charitha, C., Dutta, J. and Lalitha, C.S. (2014) Gap Functions for Vector Variational Inequalities. Optimization, 64, 1499-1520. https://doi.org/10.1080/02331934.2014.888556

[3] Bensoussan, A., Goursat, M. and Lions, J.L. (1973) Controle impulsionnel et inequations quasi-variationnelles stationnaires. Comptes Rendus de l'Académie des Sciences Paris, Series A, 276, 1279-1284.

[4] Ansari, Q.H., Lalitha, C.S. and Mehta, M. (2013) Generalized Convexity, Nonsmooth Variational Inequalities, and Nonsmooth Optimization. Chapman and Hall, Taylor \& Francis, UK.

[5] Aussel, D., Correa, R. and Marechal, M. (2011) Gap Functions for Quasivariational Inequalities and Generalized Nash Equilibrium Problems. Journal of Optimization Theory and Applications, 151, 474-488. https://doi.org/10.1007/s10957-011-9898-Z

[6] Baiocchi, C. and Capelo, A. (1984) Variational and Quasi-Variational Inequalities: Applications to Free Boundary Problems. John Wiley, Chichester.

[7] Kum, S. (1998) An Example of Generalized Quasi-Variational Inequality. Journal of Optimization Theory and Applications, 99, 253-258.

https://doi.org/10.1023/A:1021764630523

[8] Pang, J.-S. and Fukushima, M. (2005) Quasi-Variational Inequalities, Generalized Nash Equilibria, and Multi-Leader-Follower Games. Computational Management Science, 2, 21-56. https://doi.org/10.1007/s10287-004-0010-0

[9] Giannessi, F. and Maugeri, A. (2007) Variational Analysis and Applications, Nonconvex Optimization and Its Applications. Springer, Berlin. 
[10] Chen, G.Y. and Cheng, G.M. (1987) Vector Variational Inequality and Vector Optimization Problem, toward Interactive and Intelligent Decision Support Systems In: Sawaragi, Y., Inoue, K. and Nakayama, H., Eds., Lecture Notes in Economics and Mathematical Systems, Springer Berlin Heidelberg, Vol. 285, 408-416. https://doi.org/10.1007/978-3-642-46607-6_44

[11] Lee, G.M., Kim, D.S. and Lee, B.S. (1996) Generalized Vector Variational Inequality. Applied Mathematics Letters, 9, 39-42. https://doi.org/10.1016/0893-9659(95)00099-2

[12] Lee, G.M., Kim, D.S., Lee, B.S. and Cho, S.J. (1993) Generalized Vector Variational Inequality and Fuzzy Extension. Applied Mathematics Letters, 6, 47-51. https://doi.org/10.1016/0893-9659(93)90077-Z

[13] Lin, K.L., Yang, D.P. and Yao, J.C. (1997) Generalized Vector Variational Inequalities. Journal of Optimization Theory and Applications, 92, 117-125. https://doi.org/10.1023/A:1022640130410

[14] Konnov, I.V. and Yao, J.C. (1997) On the Generalized Vector Variational Inequality Problem. Journal of Mathematical Analysis and Applications, 206, 42-58. https://doi.org/10.1006/jmaa.1997.5192

[15] Daniilidis, A. and Hadjisavvas, N. (1996) Existence Theorems for Vector Variational Inequalities. Bulletin of the Australian Mathematical Society, 54, 473-481. https://doi.org/10.1017/S0004972700021882

[16] Giannessi, F. (2000) Vector Variational Inequality and Vector Equilibria: Mathematical Theories, Nonconvex Optimization and Its Applications. Kluwer Academic Publisher, Boston, 38. https://doi.org/10.1007/978-1-4613-0299-5

[17] Konnov, I.V. (2005) A Scalarization Approach for Vector Variational Inequalities with Applications. Journal of Global Optimization, 32, 517?-527. https://doi.org/10.1007/s10898-003-2688-x

[18] Gupta, R. and Mehra, A. (2012) Gap Functions and Error Bounds for Quasi Variational Inequalities. Journal of Global Optimization, 53, 737-748. https://doi.org/10.1007/s10898-011-9733-y

[19] Taji, K. (2008) On Gap Function for Quasi-Variational Inequalities. Abstract and Applied Analysis, 2008, Article ID: 531361, 7 p. http://www.emis.de/journals/HOA/AAA/Volume2008/531361.pdf

[20] Auslender, A. (1976) Optimisation: Methods Numeriques. Masson, Paris. 